\title{
Regression Models Describing Rosa hybrida Response to Day/Night Temperature and Photosynthetic Photon Flux
}

\author{
Douglas A. Hopper'and P. Allen Hammer ${ }^{2}$ \\ Department of Horticulture, Purdue University, West Lafayette, IN 47907
}

\begin{abstract}
Additional index words. central composite design, canonical analysis, light, irradiance
Abstract. A central composite rotatable design was used to estimate quadratic equations describing the relationship of irradiance, as measured by photosynthetic photon flux (PPF), and day (DT) and night (NT) temperatures to the growth and development of Rosa hybrida L. in controlled environments. Plants were subjected to 15 treatment combinations of the PPF, DT, and NT according to the coding of the design matrix. Day and night length were each 12 hours. Environmental factor ranges were chosen to include conditions representative of winter and spring commercial greenhouse production environments in the Midwestern United States. After an initial hard pinch, 11 plant growth characteristics were measured every 10 days and at flowering. Four plant characteristics were recorded to describe flower bud development. Response surface equations were displayed as three-dimensional plots, with DT and NT as the base axes and the plant character on the z-axis while PPF was held constant. Response surfaces illustrated the plant response to interactions of DT and NT, while comparisons between plots at different PPF showed the overall effect of PPF. Canonical analysis of all regression models revealed the stationary point and general shape of the response surface. All stationary points of the significant models were located outside the original design space, and all but one surface was a saddle shape. Both the plots and analysis showed greater stem diameter, as well as higher fresh and dry weights of stems, leaves, and flower buds to occur at flowering under combinations of low DT $(\leq 17 \mathrm{C})$ and low NT $(\leq 14 \mathrm{C})$. However, low DT and NT delayed both visible bud formation and development to flowering. Increased PPF increased overall flower stem quality by increasing stem diameter and the fresh and dry weights of all plant parts at flowering, as well as decreased time until visible bud formation and flowering. These results summarize measured development at flowering when the environment was kept constant throughout the entire plant growth cycle.
\end{abstract}

Growth and development of the greenhouse rose are strongly influenced by irradiance and temperature. Studies in the greenhouse (Armitage and Tsujita, 1979a, 1979b; Tsujita, 1982) and in growth chambers (Butt and Tsujita, 1986) have illustrated that flower weight increases with increasing irradiance. Yield, as flowers per square meter, also increases as irradiance increases either naturally (Post and Howland, 1946; Mattson and Widmer, 1971) or with artificial supplementation (Carpenter and Anderson, 1972; Cockshull, 1975; White and Richter, 1973). PPF, those wavelengths of radiation in the 400- to 700-nm band, have been shown to have the most direct influence on plant growth (Savage, 1982). Daily supplementation with as low as $65 \mu \mathrm{mol} \cdot \mathrm{m}^{-2} \cdot \mathrm{s}^{-1}$ for $12 \mathrm{~h}$ increased yield and quality while decreasing flowering time by 1 to 2 days during a period of five winter months from October to March (Tsujita, 1982). Supplementation with $158 \mu \mathrm{mol} \cdot \mathrm{m}^{-2} \cdot \mathrm{s}^{-1}$ for $12 \mathrm{~h}$ more than doubled the yield over nonirradiated control plants while reducing flowering time by 4 to 5 days over the 4 months from October to January (Armitage and Tsujita, 1978a).

Temperature has been shown to influence rate of plant development and flower quality. Low temperatures (10-12C) delayed development for some cultivars (DeVries et al., 1986; Hanan, 1979) and resulted in higher incidence of both blind shoots (bud atrophy) and "bullhead" development (malformed

\footnotetext{
Received for publication 13 Mar. 1990. Journal paper no. 12414 of the Purdue Univ. Agricultural Experiment Station. We thank E.G. Hill Co. for supplying the plants for this study. We gratefully acknowledge grants from the American Floral Endowment to support the PhD research of D.A.H. The cost of publishing this paper was defrayed in part by the payment of page charges. Under postal regulations, this paper therefore must be hereby marked advertisement solely to indicate this fact.

'Graduate Research Assistant. Currently, Assistant Professor, Dept. of Horticulture, Colorado State Univ., Fort Collins, CO 80523.

${ }^{2}$ Professor.
}

flowers) (Hanan, 1979; Moe, 1971). The interaction between PPF and temperature has been demonstrated to have a significant impact on rose growth (Holcomb and Arteca, 1987; Jaio et al., 1988).

Interactions between several independent environmental factors can be quantified using response surface methods (Myers, 1976). Previous plant growth studies have enlisted the central composite experimental design to produce a rigorous mathematical representation of response surfaces (Armitage et al., 1981; Hammer and Langhans, 1976; Heins et al., 1986; Kraszewski and Ormrod, 1986). A central composite rotatable design has the advantage of high efficiency when experimental units are homogeneous within a replication for a study of multiple factors, while rotatability allows uniform precision for predicted responses (Box and Draper, 1987; Box et al., 1978; Cochran and Cox, 1957; Kraszewski and Ormrod, 1986). The objective of this study was to use a central composite rotatable design to develop quadratic regression models for the response surfaces of several plant characteristics that reflect rose growth and development. Canonical analysis (Myers, 1976) facilitated simplified description of these surfaces and a better understanding of how plant response changes with movement on these surfaces within the original design space.

\section{Materials and Methods}

\section{General methods}

All treatments were conducted in Sherer Model CEC 512-37 controlled-environment chambers (Sherer-Gillett, Marshall, Mich.). The compartment dimensions were $2.6 \times 1.34 \times 1.6$

Abbreviations: DT, day temperature, NT, night temperature; PPF, photosynthetic photon flux. 
m (length $\times$ width $\times$ height), creating an interior volume of $5.57 \mathrm{~m}^{3}$. The growth bench was $2.54 \times 1.3 \mathrm{~m}$.

PPF was generated using combinations of 28, 2.44-m-long, 215-W and eight, 1.22-m-long, 115-W Sylvania Very High Output cool-white fluorescent lamps and 12, 75-W Sylvania Super Saver incandescent lamps (Sylvania Lighting Corp., Danvers, Mass.). PPF was measured throughout each treatment period using a LICOR LI-185 meter with a LI-COR LI-190S quantum (photon) sensor (LI-COR, Lincoln, Neb.). The sensor was placed on a level platform at the top of the plant canopy. There was no barrier between the lamps and plants. PPF was adjusted by altering the number of lamps and distance of the plants from the lamps.

Relative humidity (RH) was measured using a Bendix Model 566-3 Psychron aspirated psychrometer (Bendix Environmental Science Division, Baltimore). Flooding the plant compartment floor resulted in an $\mathrm{RH}$ of $50 \%$ to $95 \%$. A Sherer Transistorized

Table 1. Selected levels of environmental factors and the set points established according to the desirer matrix.

\begin{tabular}{|c|c|c|c|c|c|c|}
\hline \multirow{3}{*}{$\begin{array}{c}\text { Treatment } \\
\text { no. }\end{array}$} & & & & \multicolumn{3}{|c|}{ Environmental set point } \\
\hline & \multicolumn{3}{|c|}{ Coded axis } & PPF & \multicolumn{2}{|c|}{ Temp $\left({ }^{\circ} \mathrm{C}\right)$} \\
\hline & $\mathrm{X}_{\mathrm{PPF}}$ & $\mathrm{X}_{\mathrm{DT}}$ & $X_{\mathrm{NT}}$ & $\left(\mu \mathrm{mol} \cdot \mathrm{s}^{-1} \cdot \mathrm{m}^{-2}\right)$ & Day & Night \\
\hline 1 & -1.68 & 0 & 0 & 50 & 20 & 17 \\
\hline 2 & -1 & -1 & -1 & 100 & 17 & 14 \\
\hline 3 & -1 & -1 & 1 & 100 & 17 & 20 \\
\hline 4 & -1 & 1 & -1 & 100 & 23 & 14 \\
\hline 5 & -1 & 1 & 1 & 100 & 23 & 20 \\
\hline 6 & 0 & 0 & 0 & 175 & 20 & 17 \\
\hline 7 & 0 & 0 & -1.68 & 175 & 20 & 12 \\
\hline 8 & 0 & 0 & 1.68 & 175 & 20 & 22 \\
\hline 9 & 0 & 1.68 & 0 & 175 & 25 & 17 \\
\hline 10 & 0 & -1.68 & $\mathbf{0}$ & 175 & 15 & 17 \\
\hline 11 & 1 & -1 & 1 & 250 & 17 & 20 \\
\hline 12 & 1 & 1 & 1 & 250 & 23 & 20 \\
\hline 13 & 1 & -1 & -1 & 250 & 17 & 14 \\
\hline 14 & 1 & 1 & -1 & 250 & 23 & 14 \\
\hline 15 & 1.68 & 0 & 0 & 300 & 20 & 17 \\
\hline
\end{tabular}

${ }^{2}$ Coded values from the design matrix for the central composite rotatable design.
Electronic Dual Controller (Model-2-113) was adjusted to achieve desired set points for the day/night temperature regime of each treatment based on temperature readings from the aspirated psychrometer. Readings were taken every 7 to 14 days throughout the course of the treatment as a spot check to confirm integrity of the control system. A temperature probe in the chamber linked to the controller was shielded from direct radiation with aluminum foil.

Thirty-two XXX (highest grade) stock plants of 'Royalty' rose (E.G. Hill Co., Richmond, Ind.) were planted in $30-\mathrm{cm}-$ diameter (15 liters) round plastic pots. The growing medium was 1 soil :2 sphagnum peat :2 perlite (by volume), $\mathrm{pH} 6.2$, amended with the following nutrients (all per cubic meter): 744 $\mathrm{g}$ treble superphosphate, $496 \mathrm{~g}$ potassium nitrate, $496 \mathrm{~g}$ magnesium sulfate, $4 \mathrm{~kg}$ agricultural limestone, and $62 \mathrm{~g}$ Fritted Trace Element mix (Robert B. Peters Co., Allentown, Pa.). The plants were grown in a glass greenhouse with ambient irradiance and a 24/16C day/night cycle. Liquid fertilization with each irrigation provided $200 \mathrm{mg}$ N/liter and $200 \mathrm{mg} \mathrm{K} / \mathrm{liter}$ at $\mathrm{pH} 6.0$ by injecting $75 \%(\mathrm{v} / \mathrm{v})$ technical grade phosphoric acid into the system (29 mg P/liter). Periodic applications of monosodium ferric diethylenetriamine pentaacetate (Sequestrene 330) were made as a soil drench of $5.8 \mathrm{~g} /$ liter to prevent iron-deficiency chlorosis.

Plants used in the treatments were started as single-node cuttings from the stock plants. Cuttings were placed in a 6 sphagnum peat $: 4$ perlite : 1 vermiculite (by volume) medium, $\mathrm{pH}$ 5.5 , filling $6.5 \times 9.0 \times 6.0-\mathrm{cm}$ single cells of 24 per $27 \times$ $54 \times 6.0-\mathrm{cm}$ flat. Cutting size varied in stem diameter $(0.5-$ $1.2 \mathrm{~cm})$, stem length $(3.0-10.0 \mathrm{~cm})$, and in leaf blade length $(8.0-16.0 \mathrm{~cm})$. The basal end of the cutting was dipped in a commercial, powdered preparation (Hormo-Root 2; Hortus Products Co., Newfoundland, N.J.) of 2\% indole-3-butyric acid (IBA) before placement in the medium.

The cuttings were held under intermittent mist (4 sec every 4 min during daylight) for 4 weeks at a constant 21C and ambient irradiance. During the study, a commercial antifungal preparation of 1 bromo-3-chloro-5,5 dimethyl-2,4-imidazolidinedione (Agribrom; Great Lakes Chemical Corp., West Lafayette, Ind.) was added to the mist system via an injector; this

Table 2. Terms in five, full quadratic models of plant characteristics and model significance. The model form is: $\mathrm{y}=b_{o}+b_{1} \mathrm{X}_{\mathrm{PPF}}+b_{2} \mathrm{X}_{\mathrm{DT}}$ $+b{ }_{3} \mathrm{X}_{\mathrm{NT}}+b{ }_{11} \mathrm{X}_{\mathrm{PPF}}^{2}+b{ }_{22} \mathrm{X}_{\mathrm{DT}}^{2}+b_{33} \mathrm{X}_{\mathrm{NT}}^{2}+b{ }_{12} \mathrm{X}_{\mathrm{PPF}} \mathrm{X}_{\mathrm{DT}}+b{ }_{13} \mathrm{X}_{\mathrm{PPF}} \mathrm{X}_{\mathrm{NT}}+b_{23} \mathrm{X}_{\mathrm{DT}} \mathrm{X}_{\mathrm{NT}}$.

\begin{tabular}{|c|c|c|c|c|c|c|c|c|c|c|c|}
\hline \multirow[b]{4}{*}{ Coefficient } & \multirow[b]{4}{*}{ Factor $^{\mathbf{z}}$} & \multicolumn{10}{|c|}{ Characteristic } \\
\hline & & & & & & & Fre & & & & \\
\hline & & \multicolumn{2}{|c|}{ Stem diam } & \multicolumn{2}{|c|}{ Stem } & \multicolumn{2}{|c|}{ Leaf } & \multicolumn{2}{|c|}{ Flower } & \multicolumn{2}{|c|}{ Total } \\
\hline & & Estimate & $\mathbf{s}_{\mathrm{b}}$ & Estimate & $\mathbf{s}_{\mathrm{b}}$ & Estimate & $\mathbf{s}_{\mathrm{b}}$ & Estimate & $s_{b}$ & Estimate & $\mathbf{s}_{\mathrm{b}}$ \\
\hline$\overline{b_{0}}$ & 1 & 4.95 & 0.152 & 5.72 & 0.380 & 11.0 & 0.561 & 10.3 & 1.12 & 27.0 & 1.89 \\
\hline$b_{1}$ & $\mathrm{X}_{\mathrm{PPF}}$ & 0.638 & 0.0926 & 1.68 & 0.231 & 2.73 & 0.341 & 2.44 & 0.680 & 6.84 & 1.15 \\
\hline$b_{2}$ & $\mathrm{X}_{\mathrm{DT}}$ & -0.313 & 0.0926 & -1.08 & 0.231 & -2.55 & 0.341 & -2.06 & 0.680 & -5.70 & 1.15 \\
\hline$b_{3}$ & $\mathrm{X}_{\mathrm{NT}}$ & -0.126 & 0.0926 & -0.819 & 0.231 & -1.28 & 0.341 & -1.97 & 0.680 & -4.07 & 1.15 \\
\hline$b_{11}^{3}$ & $X_{P P F} \times X_{P P F}$ & 0.0181 & 0.0935 & 0.159 & 0.233 & -0.0816 & 0.345 & -0.756 & 0.687 & -0.679 & 1.16 \\
\hline$b_{12}$ & $X_{\mathrm{PPF}} \times \mathrm{X}_{\mathrm{DT}}$ & 0.0841 & 0.121 & -0.000988 & 0.301 & -0.771 & 0.444 & 0.0562 & 0.885 & -0.716 & 1.50 \\
\hline$b_{22}$ & $\mathrm{X}_{\mathrm{DT}} \times \mathrm{X}_{\mathrm{DT}}$ & 0.0693 & 0.0935 & 0.0448 & 0.233 & 0.299 & 0.345 & 0.145 & 0.687 & 0.489 & 1.16 \\
\hline$b_{13}$ & $\mathrm{X}_{\mathrm{PPF}} \times \mathrm{X}_{\mathrm{NT}}$ & -0.0349 & 0.121 & -0.162 & 0.301 & -0.0712 & 0.444 & 0.0634 & 0.885 & -0.170 & 1.50 \\
\hline$b_{23}$ & $\mathrm{X}_{\mathrm{DT}} \times \mathrm{X}_{\mathrm{NT}}$ & -0.211 & 0.121 & -0.0475 & 0.301 & 0.0471 & 0.444 & $-0: 280$ & 0.885 & -0.280 & 1.50 \\
\hline & $X_{\mathrm{NT}} \times X_{\mathrm{NT}}$ & 0.027 & 0.0935 & 0.426 & 0.233 & 0.329 & 0.345 & 0.801 & 0.687 & 1.56 & 1.16 \\
\hline Significance & -- & & & $* * *$ & & $* * *$ & & * & & $* *$ & \\
\hline$R^{2}$ & -- & & & 0.910 & & 0.93 & & 0.7 & & 0.89 & \\
\hline
\end{tabular}

${ }^{\mathrm{z}}$ As shown in the design matrix in Table $1 . \mathrm{X}_{\mathrm{PPF}}=$ coded photosynthetic photon flux; $\mathrm{X}_{\mathrm{DT}}=$ coded day temperature; $\mathrm{X}_{\mathrm{NT}}=$ coded night temperature.

$* * * * * *$ Significantly different at $P=0.05,0.01$, or 0.001 , respectively. 
Table 3. Terms in four, full quadratic models and model significance for plant dry weight characteristics. The model form is: $\mathrm{y}=b_{0}+b_{l}$ $\mathrm{X}_{\mathrm{PPF}}+b_{2} \mathrm{X}_{\mathrm{DT}}+b_{3} \mathrm{X}_{\mathrm{NT}}+b_{11} \mathrm{X}_{\mathrm{PPF}}^{2} b_{22} X^{2}{ }_{\mathrm{DT}}+b_{33} \mathrm{X}_{\mathrm{NT}}^{2}+b_{12} \mathrm{X}_{\mathrm{PPF}} \mathrm{X}_{\mathrm{DT}}+b_{3} \mathrm{X}_{\mathrm{PPF}} \mathrm{X}_{\mathrm{NT}}+b_{23} \mathrm{X}_{\mathrm{DT}} \mathrm{X}_{\mathrm{NT}}$.

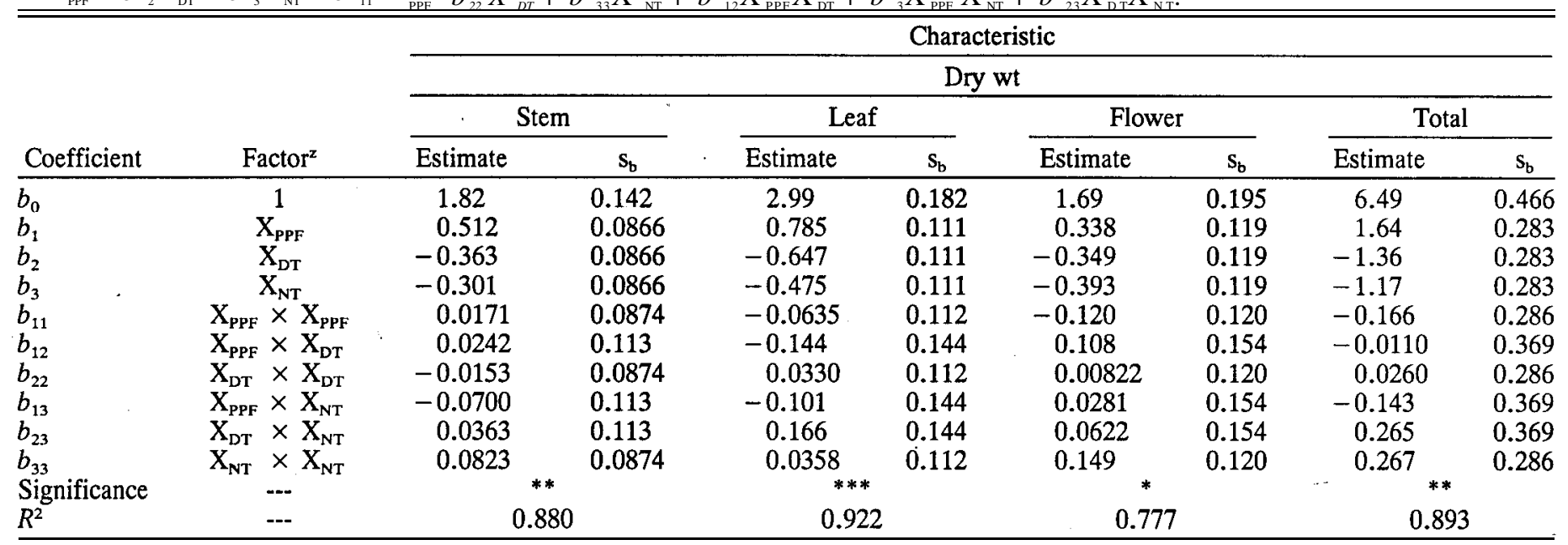

${ }^{\bar{z}}$ As shown in the design matrix in Table $1 . \mathrm{X}_{\mathrm{PPF}}=$ coded photosynthetic photon flux; $\mathrm{X}_{\mathrm{DT}}=$ coded day temperature; $\mathrm{X}_{\mathrm{NT}}=$ coded night temperature.

*,**,*** Significantly different at $P=0.05,0.01$, or 0.001 , respectively.

Table 4. Terms in four, full quadratic models for flower development and model significance. The model form is: $\mathrm{y}=b_{0}+b_{1} X_{P P F}+b_{2} X_{D T}$ $+b{ }_{3} \mathrm{X}_{\mathrm{NT}}+b{ }_{11} \mathrm{X}_{\mathrm{PPF}}^{2}+b{ }_{22} \mathrm{X}_{\mathrm{DT}}^{2}+b_{33} \mathrm{X}_{\mathrm{NT}}^{2}+b{ }_{12} \mathrm{X}_{\mathrm{PPF}} \mathrm{X}_{\mathrm{DT}}+b{ }_{13} \mathrm{X}_{\mathrm{PPF}} \mathrm{X}_{\mathrm{NT}}+b{ }_{23} \mathrm{X}_{\mathrm{DT}} \mathrm{X}_{\mathrm{NT}}$.

\begin{tabular}{|c|c|c|c|c|c|c|c|c|c|}
\hline \multirow[b]{4}{*}{ Coefficient } & \multirow[b]{4}{*}{ Factor $^{\mathbf{z}}$} & \multicolumn{8}{|c|}{ Characteristic } \\
\hline & & \multicolumn{3}{|c|}{${ }^{\prime}$} & \multicolumn{3}{|c|}{ Interval (days) to } & & \\
\hline & & \multicolumn{2}{|c|}{ Visible bud } & \multicolumn{2}{|c|}{ First color } & \multicolumn{2}{|c|}{ Sepal reflex } & \multicolumn{2}{|c|}{ Flower } \\
\hline & & Estimate & $s_{b}$ & Estimate & $s_{b}$ & Estimate & $s_{\mathrm{b}}$ & Estimate & $s_{b}$ \\
\hline$b_{0}$ & 1 & 25.1 & 0.252 & 41.3 & 0.426 & 51.9 & 0.501 & 52.8 & 0.406 \\
\hline$b_{1}$ & $\mathrm{X}_{\mathrm{PPF}}$ & -1.40 & 0.153 & -2.98 & 0.259 & -3.12 & 0.305 & -3.41 & 0.247 \\
\hline$b_{2}$ & $\mathrm{X}_{\mathrm{DT}}$ & -4.17 & 0.153 & -5.57 & 0.259 & -7.03 & 0.305 & -8.24 & 0.247 \\
\hline$b_{3}$ & $\mathrm{X}_{\mathrm{NT}}$ & -2.90 & 0.153 & -4.09 & 0.259 & -4.99 & 0.305 & -5.32 & 0.247 \\
\hline$b_{11}$ & $\mathbf{X}_{\mathrm{PPF}} \times \mathrm{X}_{\mathrm{PPF}}$ & 0.248 & 0.155 & 0.740 & 0.262 & -0.543 & 0.308 & -0.319 & 0.249 \\
\hline$b_{12}$ & $\mathbf{X}_{\mathrm{PPF}} \times \mathbf{X}_{\mathrm{DT}}$ & 0.238 & 0.199 & 0.450 & 0.337 & 0.425 & 0.397 & 0.575 & 0.322 \\
\hline$b_{22}$ & $\mathrm{X}_{\mathrm{DT}} \times \mathrm{X}_{\mathrm{DT}}$ & 1.00 & 0.155 & 1.12 & 0.262 & 0.105 & 0.308 & 1.01 & 0.249 \\
\hline$b_{13}$ & $X_{\mathrm{PPF}} \times \mathrm{X}_{\mathrm{NT}}$ & -0.238 & 0.199 & -0.275 & 0.337 & 0.0750 & 0.397 & 0.325 & 0.322 \\
\hline$b_{23}$ & $\mathrm{X}_{\mathrm{DT}} \times \mathrm{X}_{\mathrm{NT}}$ & 0.913 & 0.199 & 1.35 & 0.337 & 0.8750 & 0.397 & 0.725 & 0.322 \\
\hline & $\mathrm{X}_{\mathrm{NT}} \times \mathrm{X}_{\mathrm{NT}}$ & -0.274 & 0.155 & -0.160 & 0.262 & 0.0332 & 0.308 & -0.211 & 0.249 \\
\hline Significance & -.. & & & & & ** & & & \\
\hline$R^{2}$ & --- & & & 0.9 & & 0.9 & & 0.5 & \\
\hline
\end{tabular}

${ }^{z}$ As shown in the design matrix in Table $1 . \mathrm{X}_{\mathrm{PPF}}=$ coded photosynthetic photon flux; $\mathrm{X}_{\mathrm{DT}}=$ coded day temperature; $\mathrm{X}_{\mathrm{NT}}=$ coded night temperature.

$*, * * * * * ! \quad$ Significantly different at $P=0.05,0.01$, or 0.001 , respectively.

prevented algae accumulation and improved overall cleanliness. After 4 weeks, the rooted cuttings were transplanted into 10$\mathrm{cm}$, square plastic pots $(640 \mathrm{ml})$ of growing medium as was described for the stock plants, and mist was gradually reduced over 1 week before moving the plants to a growth chamber for treatment. Plants were acclimated to the growth chamber treatment conditions for 7 to 10 days before hard-pinching to leave two five-leaflet leaves on each new plant shoot. At the time of pinching, the 50 most uniform plants were selected for treatment, tagged, and randomized within the chamber.

Daylength was $12 \mathrm{~h}$ in all treatments. Day encompassed 1130 to $2330 \mathrm{HR}$ for chamber 1 and 1200 to $2400 \mathrm{HR}$ for chamber 2; this enabled convenient monitoring of day and night regimes. The 7- to 10-day adjustment period before pinch and start of the treatment allowed diurnal rhythms to adjust to the artificial daylength. Fertilizer was applied in daily irrigations with an automatic timeclock-regulated system. Two time clocks ( $24 \mathrm{~h}$ and $30 \mathrm{~min}$ ) wired in series allowed 5 to $10 \mathrm{~min}$ of watering for a $15-\mathrm{min}$ period each day, depending on plant age and water requirements. The clocks engaged a submersible pump that supplied nutrient solution from a 114-liter plastic reservoir through a spaghetti-tube, nonrecirculating irrigation system, with two lead-weighted tubes in each pot. Each liter of fertilizer solution supplied $250 \mathrm{mg}$ each of nitrogen and potassium and other essential elements by including the following: $2.45 \mathrm{~g} \mathrm{KNO}_{3}, 1.74 \mathrm{~g} \mathrm{NH}_{4} \mathrm{NO}_{3}, 0.02 \mathrm{~g} \mathrm{MgSO}_{4}, 0.02 \mathrm{~g}$ Soluble Trace Element Mix (Robert B. Peters Co.), and $18.9 \mathrm{ml}$ of $25 \mathrm{ml} 75 \%$ technical grade $\mathrm{H}_{3} \mathrm{PO}_{4}$. Every 10 to 14 days, additional iron was supplied in the chelated form, Sequestrene 330, as a soil drench of $5.8 \mathrm{~g} \cdot$ liter $^{-1}$.

Every 10 days, starting from the day of pinch, the growth from the topmost node was measured by destructive sampling of 10 plants until day 40 and at flowering. Additional shoots below the topmost node were removed after 10 days from pinch. Plant characteristics measured included number of nodes (NN), stem diameter, stem length, fresh weights of stem, leaves, and flower bud, as well as dry weights of stem, leaves, and flower 


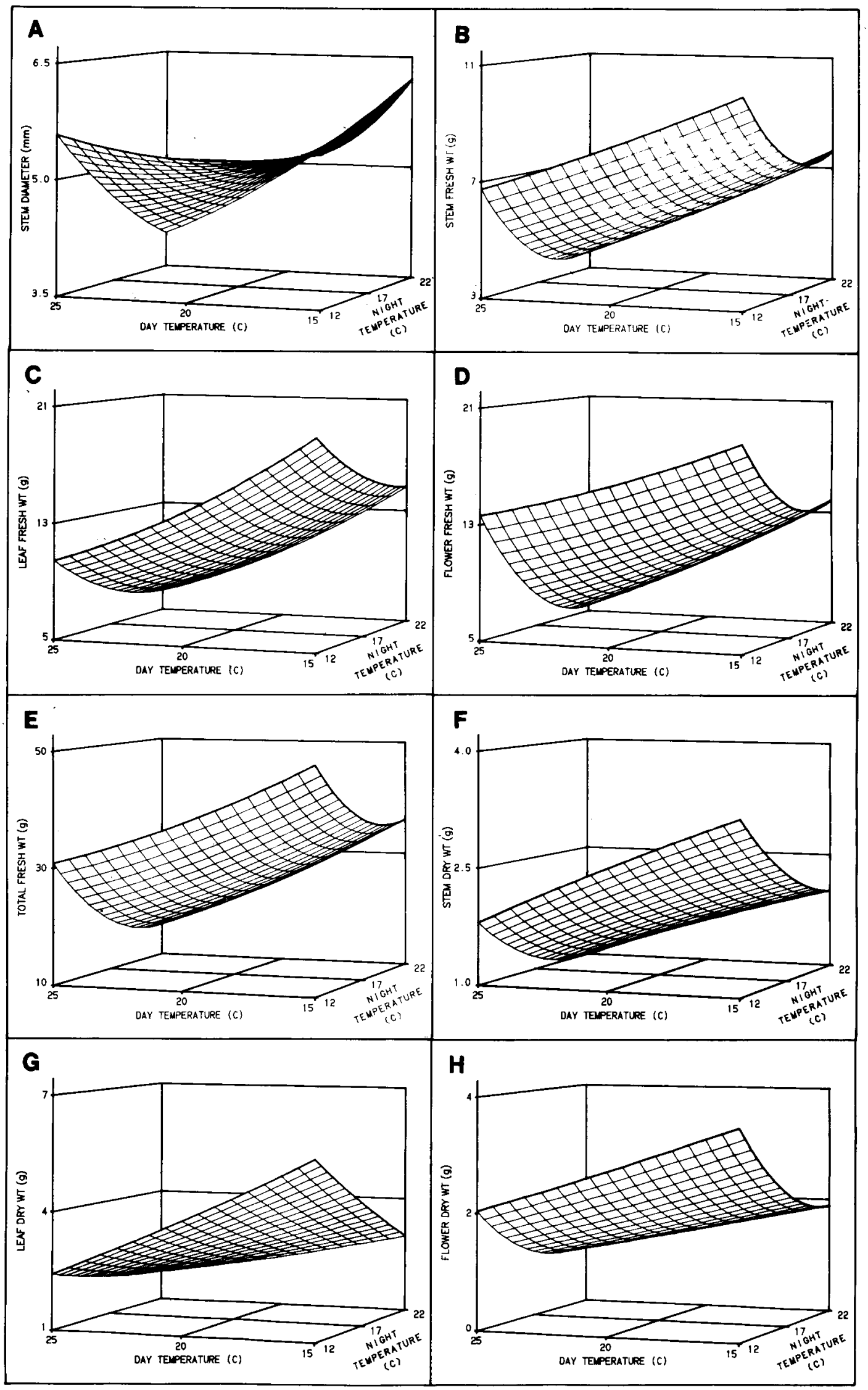

Fig. 1. Response surfaces for significant response characteristics of Rosa hybrida at flowering under constant controlled environments. Plots made for PPF $=175 \mu \mathrm{mol} \cdot \mathrm{m}^{-2} \cdot \mathrm{s}^{-1}$ (A) Stem diameter, (B) stem fresh weight, (C) leaf fresh weight, (D) flower fresh weight, (E) total fresh weight, (F) stem dry weight, $(\mathrm{G})$ leaf dry weight, (H) flower dry weight, (I) total dry weight, (J) days to visible bud, (K) days to first bud color, (L) days to sepal reflex, (M) days to flower. Fig. 1 continued next page. 


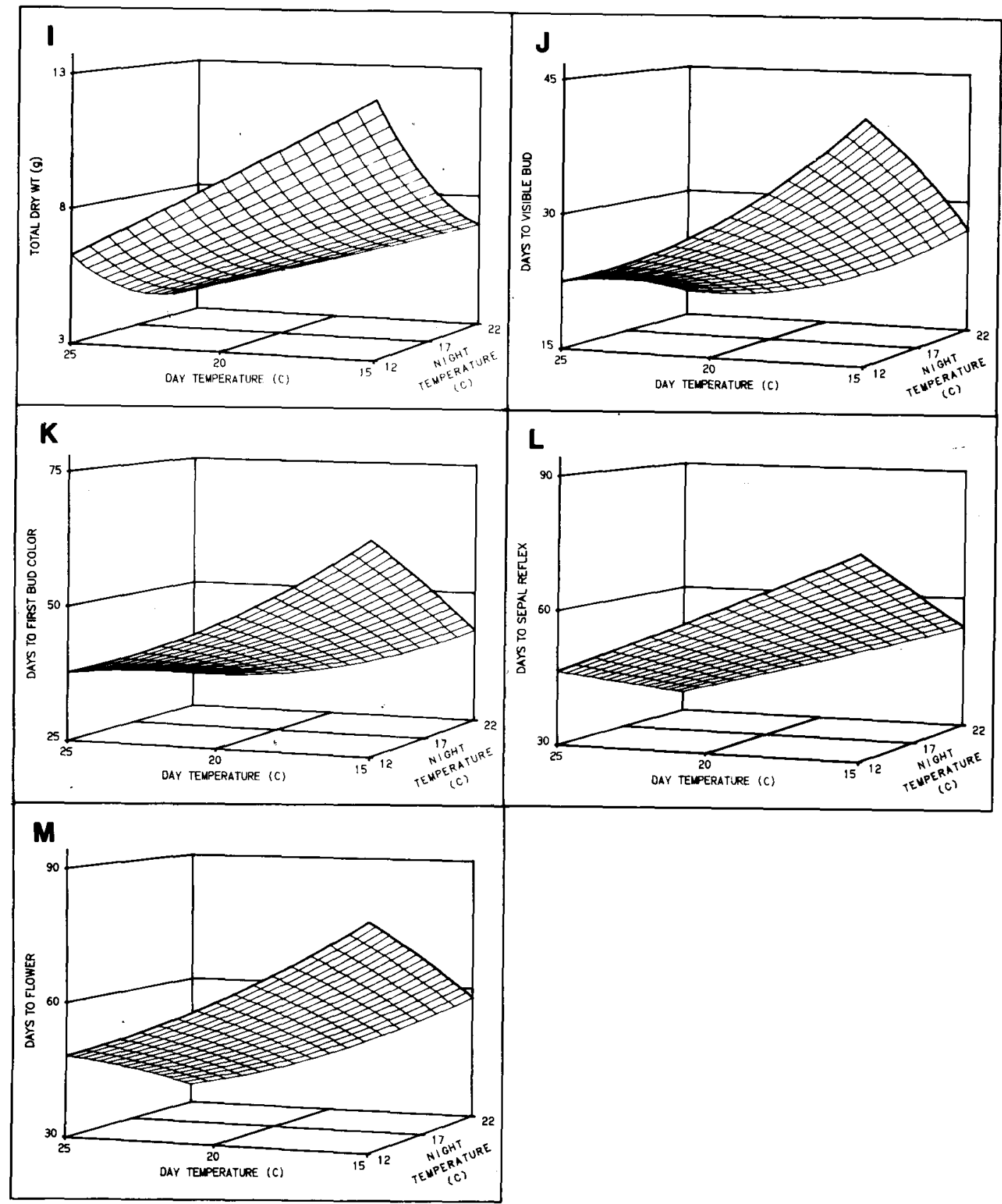

Fig. 1. Cont.

bud. Flower development was measured for each plant by recording days from pinch until visible bud, first bud color, sepals were reflexed at $90^{\circ}$ from main stem axis, and until flowering.

\section{Statistical approach}

Treatment combinations of PPF, DT, and NT were selected according to the central composite rotatable experimental design (Myers, 1976) (Table 1). The center point (treatment 6) was repeated five times to estimate experimental error. Treatments were randomly selected to be conducted in one of two growth chambers; treatments were not blocked.

Second-order polynomial equations were fit to the data by SAS data analysis (SAS, Cary, N. C.) using linear, quadratic, and cross-product terms of $\mathrm{X}_{\mathrm{PPF}}, \mathrm{X}_{\mathrm{DT}}$, and $\mathrm{X}_{\mathrm{NT}}$ (coded values of PPF, DT, and NT) to predict values of the plant characteristics at time of flowering. The higher-order interactions were considered nonsignificant and were not included in the model. These equations allowed response surfaces to be generated for each characteristic. Canonical analysis was performed on each equation by the SAS procedure RSREG. The stationary point $\left(\mathrm{x}_{0}\right)$, eigenvalues, $(\boldsymbol{\lambda})$, and the orthogonal matrix of rotation (M) were determined as described by Myers (1976).

The response surface was then rewritten in the canonical form:

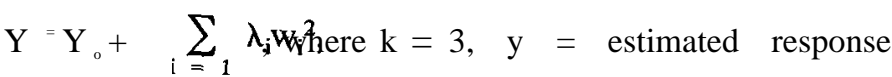
at some set of $\mathrm{x}$ vector values, $\mathrm{y}_{0}=$ estimated response at the stationary point $\mathrm{x}_{0}$ vector, $\lambda_{\mathrm{i}}=$ eigenvalue (characteristic root) of the matrix containing the coefficients of the quadratic and cross-product terms of the quadratic fitted response surface equation, and $\mathrm{w}_{\mathrm{i}}=$ component of the response in the direction of the new $\mathrm{w}_{\mathrm{i}}$-axis.

The M matrix transforms from the original $\mathrm{x}$-coordinate sys- 
Table 5. Coded and actual values for the stationary point for the canonical form, along with predicted values at the stationary point, and the associated eigenvalues and eigenvectors.

\begin{tabular}{|c|c|c|c|c|c|c|c|c|c|c|c|c|}
\hline \multirow{4}{*}{$\frac{\text { Characteristic }}{\text { Node no. }}$} & \multicolumn{6}{|c|}{ Stationary point values ${ }^{2}$} & \multirow{3}{*}{$\begin{array}{c}\text { Stationary } \\
\text { point }\end{array}$} & \multirow{3}{*}{\multicolumn{2}{|c|}{ Eigenvalues }} & \multirow{3}{*}{\multicolumn{3}{|c|}{ Eigenvectors ( $\mathrm{M}$ matrix) }} \\
\hline & \multirow{3}{*}{$\frac{X_{\mathrm{PPF}}}{2.57}$} & \multicolumn{2}{|c|}{ Coded } & \multicolumn{3}{|c|}{ Actual } & & & & & & \\
\hline & & $\mathrm{X}_{\mathrm{DT}}$ & $\mathrm{X}_{\mathrm{NT}}$ & PPF & DT & NT & & & & & & \\
\hline & & 0.520 & 1.24 & 368 & 21.6 & $2 n .7$ & 11.5 & $\begin{array}{l}\lambda_{1}= \\
\lambda_{2}= \\
\lambda_{3}=\end{array}$ & $\begin{array}{l}=0.1048 \\
=-0.08221 \\
=-0.1612\end{array}$ & $\begin{array}{r}-0.3042 \\
0.6787 \\
0.6684\end{array}$ & $\begin{array}{l}0.09025 \\
0.7191 \\
-0.6891\end{array}$ & $\begin{array}{l}0.9483 \\
0.1493 \\
0.2800\end{array}$ \\
\hline Stem diam & -22.3 & -3.12 & -12.4 & -1500 & 10.6 & -20.2 & -0.888 & $\begin{array}{l}\lambda_{1}= \\
\lambda_{2}= \\
\lambda_{3}=\end{array}$ & $\begin{array}{ll}= & 0.1782 \\
= & 0.01089 \\
= & -0.04901\end{array}$ & $\begin{array}{r}0.2595 \\
0.9304 \\
-0.2589\end{array}$ & $\begin{array}{l}0.7214 \\
-0.008552 \\
0.6924\end{array}$ & $\begin{array}{r}-0.6420 \\
0.3664 \\
0.6734\end{array}$ \\
\hline Stem length & 0.693 & -0.143 & 0.128 & 227 & 19.6 & 17.4 & 53.8 & $\begin{array}{l}\lambda_{1}= \\
\lambda_{2}= \\
\lambda_{3}=\end{array}$ & $\begin{array}{l}=\quad 2.476 \\
=-0.3434 \\
=-2.058\end{array}$ & $\begin{array}{c}-0.06594 \\
0.3739 \\
0.9251\end{array}$ & $\begin{array}{r}-0.2277 \\
0.8970 \\
-0.3788\end{array}$ & $\begin{array}{c}0.9715 \\
0.2357 \\
-0.02599\end{array}$ \\
\hline Stem fresh wt & -4.87 & 12.4 & 0.727 & -190 & 57.2 & 19.2 & -5.40 & $\begin{array}{l}\lambda_{1}= \\
\lambda_{2}= \\
\lambda_{3}=\end{array}$ & $\begin{array}{ll}= & 0.4502 \\
= & 0.1366 \\
= & 0.04305\end{array}$ & $\begin{array}{c}-0.2671 \\
0.9621 \\
0.05568\end{array}$ & $\begin{array}{c}-0.05605 \\
-0.07319 \\
0.9957\end{array}$ & $\begin{array}{l}0.9620 \\
0.2628 \\
0.07347\end{array}$ \\
\hline Leaf fresh wt & -0.498 & 3.49 & 1.64 & 138 & 30.5 & 21.9 & 4.79 & $\begin{array}{l}\lambda_{1}= \\
\lambda_{2}= \\
\lambda_{3}=\end{array}$ & $\begin{array}{rr}= & 0.5460 \\
= & 0.3227 \\
= & -0.3218\end{array}$ & $\begin{array}{c}-0.5226 \\
0.06998 \\
0.8497\end{array}$ & $\begin{array}{r}0.8341 \\
-0.1641 \\
0.5266\end{array}$ & $\begin{array}{l}0.1763 \\
0.9840 \\
0.02741\end{array}$ \\
\hline Flower fresh wt & 2.08 & 9.41 & 2.79 & 331 & 48.2 & 25.4 & 0.381 & $\begin{array}{l}\lambda_{1}= \\
\lambda_{2}= \\
\lambda_{3}=\end{array}$ & $\begin{array}{r}=0.8304 \\
=\quad 0.1177 \\
=-0.7581\end{array}$ & $\begin{array}{l}0.01605 \\
0.03869 \\
0.9991\end{array}$ & $\begin{array}{c}-0.1992 \\
0.9793 \\
-0.03473\end{array}$ & $\begin{array}{c}0.9798 \\
0.1984 \\
-0.02343\end{array}$ \\
\hline Total fresh wt & 1.02 & 7.14 & 2.01 & 252 & 41.4 & 23.0 & 6.04 & $\begin{array}{l}\lambda_{3} \\
\lambda_{1}= \\
\lambda_{2}= \\
\lambda_{3}=\end{array}$ & $\begin{array}{cc}= & 1.576 \\
= & 0.5774 \\
= & -0.7864\end{array}$ & $\begin{array}{c}-0.01797 \\
-0.2793 \\
0.9600\end{array}$ & $\begin{array}{c}-0.1220 \\
0.9536 \\
0.2752\end{array}$ & $\begin{array}{l}0.9924 \\
0.1121 \\
0.05120\end{array}$ \\
\hline Stem dry wt & -1.76 & -9.50 & 3.18 & 43 & -8.50 & 26.5 & 2.61 & $\begin{array}{l}\lambda_{1}= \\
\lambda_{2}= \\
\lambda_{3}=\end{array}$ & $\begin{array}{r}=0.09879 \\
=\quad 0.01309 \\
=-0.02772\end{array}$ & $\begin{array}{r}-0.3786 \\
0.8094 \\
-0.4489\end{array}$ & $\begin{array}{l}0.1061 \\
0.5198 \\
0.8477\end{array}$ & $\begin{array}{r}0.9195 \\
0.2733 \\
-0.2826\end{array}$ \\
\hline Leaf dry wt & 0.990 & 1.71 & 4.07 & 249 & 25.1 & 29.2 & 1.85 & $\begin{array}{l}\lambda_{1}= \\
\lambda_{2}= \\
\lambda_{3}=\end{array}$ & $\begin{array}{l}=0.1523 \\
=-0.04486 \\
=-0.1021\end{array}$ & $\begin{array}{r}-0.3737 \\
0.2326 \\
0.8979\end{array}$ & $\begin{array}{r}0.6708 \\
-0.6008 \\
0.4348\end{array}$ & $\begin{array}{l}0.6406 \\
0.7648 \\
0.06846\end{array}$ \\
\hline Flower dry wt & 2.52 & 2.31 & 0.597 & 364 & 26.9 & 18.8 & 1.60 & $\begin{array}{l}\lambda_{1}= \\
\lambda_{2}= \\
\lambda_{3}=\end{array}$ & $\begin{array}{ll}= & 0.1583 \\
= & 0.01918 \\
= & -0.1397\end{array}$ & $\begin{array}{l}0.09453 \\
0.3290 \\
0.9396\end{array}$ & $\begin{array}{r}0.2346 \\
0.9099 \\
-0.3422\end{array}$ & $\begin{array}{l}0.9675 \\
-0.2528 \\
-0.008806\end{array}$ \\
\hline Total dry wt & 2.24 & -8.20 & 6.84 & 343 & -4.60 & 37.5 & 9.90 & $\begin{array}{l}\lambda_{1}= \\
\lambda_{2}= \\
\lambda_{3}=\end{array}$ & $\begin{array}{l}=0.3350 \\
=-0.02857 \\
=-0.1792\end{array}$ & $\begin{array}{r}-0.1345 \\
0.1565 \\
0.9785\end{array}$ & $\begin{array}{c}0.3920 \\
0.9153 \\
-0.09254\end{array}$ & $\begin{array}{r}0.9101 \\
-0.3711 \\
0.1845\end{array}$ \\
\hline Days to visible bud & 0.926 & 2.59 & -1.37 & 244 & 27.8 & 12.9 & 21.1 & $\begin{array}{l}\lambda_{1}= \\
\lambda_{2}= \\
\lambda_{3}=\end{array}$ & $\begin{array}{l}=1.156 \\
=\quad 0.2729 \\
=-0.4527\end{array}$ & $\begin{array}{l}0.08559 \\
0.9740 \\
0.2096\end{array}$ & $\begin{array}{l}0.9513 \\
-0.01734 \\
-0.3079\end{array}$ & $\begin{array}{r}0.2962 \\
-0.2258 \\
0.9281\end{array}$ \\
\hline Days to first color & 0.912 & 2.96 & -1.08 & 243 & 28.9 & 13.8 & 33.9 & $\begin{array}{l}\lambda_{1}= \\
\lambda_{2}= \\
\lambda_{3}=\end{array}$ & $\begin{array}{c}=1.443 \\
=\quad 0.7438 \\
=-0.4887\end{array}$ & $\begin{array}{l}0.2191 \\
0.9600 \\
0.1742\end{array}$ & $\begin{array}{r}0.9058 \\
-0.1337 \\
-0.4021\end{array}$ & $\begin{array}{r}0.3627 \\
-0.2459 \\
0.8989\end{array}$ \\
\hline Days to sepal reflex & -0.355 & 5.20 & 6.96 & 148 & 35.6 & 37.9 & 16.8 & $\begin{array}{l}\lambda_{3} \\
\lambda_{1}= \\
\lambda_{2}= \\
\lambda_{3}=\end{array}$ & $=\begin{array}{r}0.5391 \\
=-0.3185 \\
=-0.6250\end{array}$ & $\begin{array}{l}0.1678 \\
0.4015 \\
0.9003\end{array}$ & $\begin{array}{r}0.7395 \\
0.5527 \\
-0.3843\end{array}$ & $\begin{array}{r}0.6519 \\
-0.7303 \\
0.2042\end{array}$ \\
\hline Days to flower & -1.91 & 5.97 & -3.83 & 31.8 & 37.9 & 5.51 & 41.7 & & $\begin{array}{c}= \\
=-1.181 \\
=-0.2607 \\
-0.4364\end{array}$ & $\begin{array}{l}0.2094 \\
0.5165 \\
0.8303 \\
\end{array}$ & $\begin{array}{c}0.9400 \\
-0.3401 \\
-0.02544 \\
\end{array}$ & $\begin{array}{r}0.2693 \\
0.7858 \\
-0.5567 \\
\end{array}$ \\
\hline
\end{tabular}

${ }^{7}$ Conversion between coded and actual values by the formulas: $\mathrm{PPF}=175+75 \times \mathrm{X}_{\mathrm{PPF}} ; \mathrm{DT}=20+3 \times \mathrm{X}_{\mathrm{DT}} ; \mathrm{NT}=17+3 \times \mathrm{X}_{\mathrm{NT}}$.

tem to a new w-coordinate system (see Table 5). The columns of the $\mathrm{M}$ matrix are eigenvectors corresponding to the eigenvalues determined by the analysis. Canonical analysis determined the nature of the stationary point (the $\mathrm{x}_{0}$ vector combination of $x$ values that defines where the slope of the response surface is zero for all dimensions) and of the response surface simply by observing sign and magnitude of the A values (Myers, 1976).

\section{Results and Discussion}

The full quadratic regression models based on the means of 10 subsamples from each treatment were significant for all plant characteristics measured, except node number and stem length (Tables 2-4). The high percentage of variability explained by each regression model was indicated by high $R^{2}$ values, all of which were above 0.75 for the significant models. Models fit for time of flower development were highly significant and had $R^{2}$ values exceeding 0.99 (Table 4 ). The response surface for stem diameter revealed small-diameter stems occurred under both high DT and NT (Fig. 1A), while large-diameter stems were promoted if low DT (15C) was used in combination with high NT (22C). Stem diameter was also enhanced by increased $\mathrm{PPF}$, since a response surface corresponding to the highest PPF 


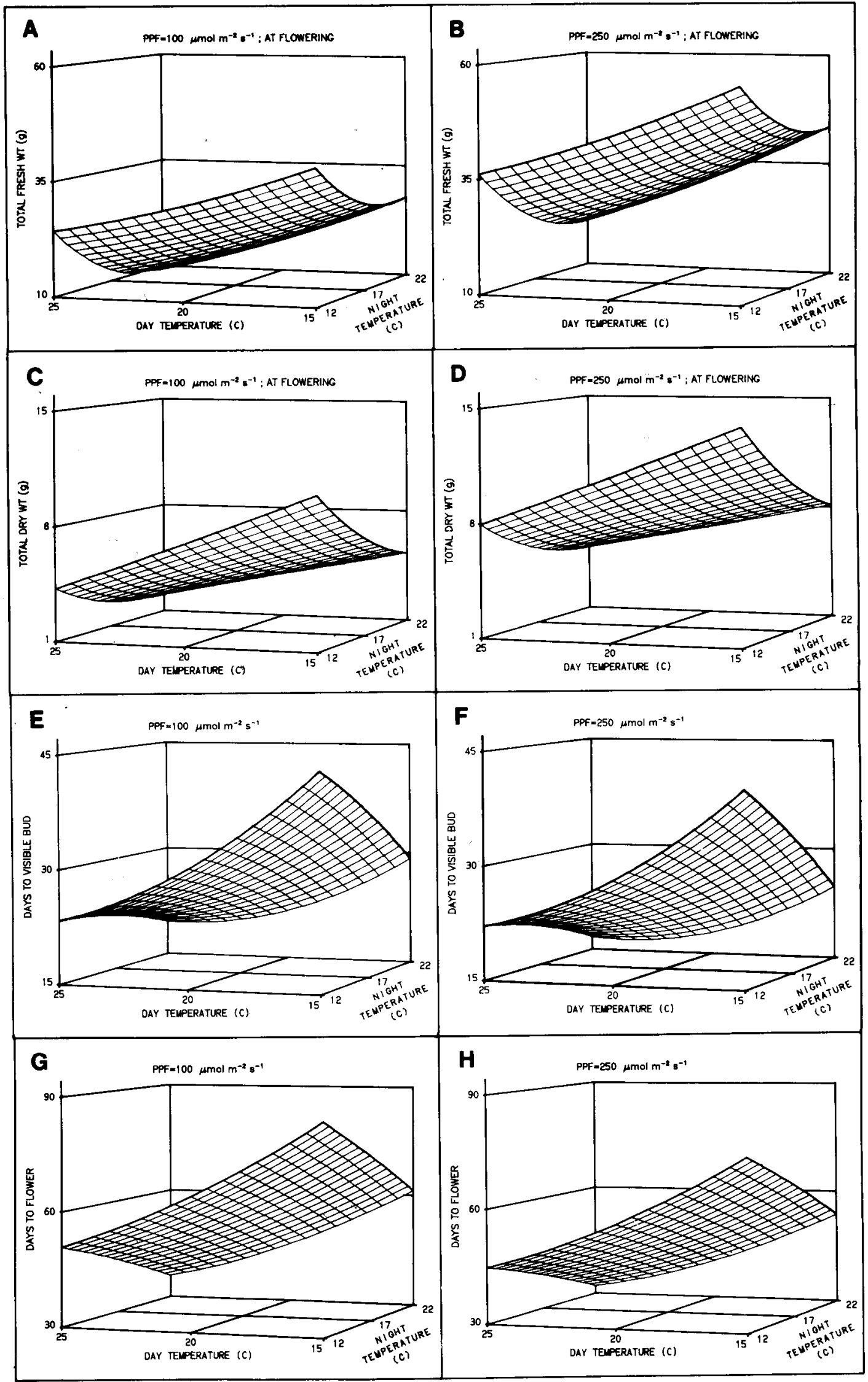

Fig. 2. Response surfaces for four plant characteristics of Rosa hybrida at flowering for two selected PPF levels under constant controlled environment conditions over the time of development. (A) Total fresh weight for PPF $=100 \mu \mathrm{mol}^{-2} \mathrm{~m}^{-2} \cdot \mathrm{s}^{-1}$ (B) total fresh weight for PPF $=250 \mu \mathrm{mol} \cdot \mathrm{m}^{-2} \cdot \mathrm{s}^{-1}$ (C) total dry weight for PPF $=100 \mu \mathrm{mol} \cdot \mathrm{m}^{-2} \cdot \mathrm{s}^{-1},(\mathbf{D})$ total dry weight for PPF $=250 \mu \mathrm{mol} \cdot \mathrm{m}^{-2} \mathrm{~s}^{-1},(\mathbf{E}) \mathrm{days}$ to visible bud for PPF $=100 \mu \mathrm{mol} \cdot \mathrm{m}^{-2} \cdot \mathrm{s}^{-1},(\mathbf{F})$ days to visible bud for PPF $=250 \mu \mathrm{mol} \cdot \mathrm{m}^{-2} \cdot \mathrm{s}^{-1}$, (G) days to flower for PPF $=100$ $\left.\mu \mathrm{mol} \cdot \mathrm{m}^{-2} \cdot \mathrm{s}^{-1}, \mathbf{H}\right)$ days to flower for $\mathrm{PPF}=250 \mu \mathrm{mol} \cdot \mathrm{m}^{-2} \cdot \mathrm{s}^{-1}$. 


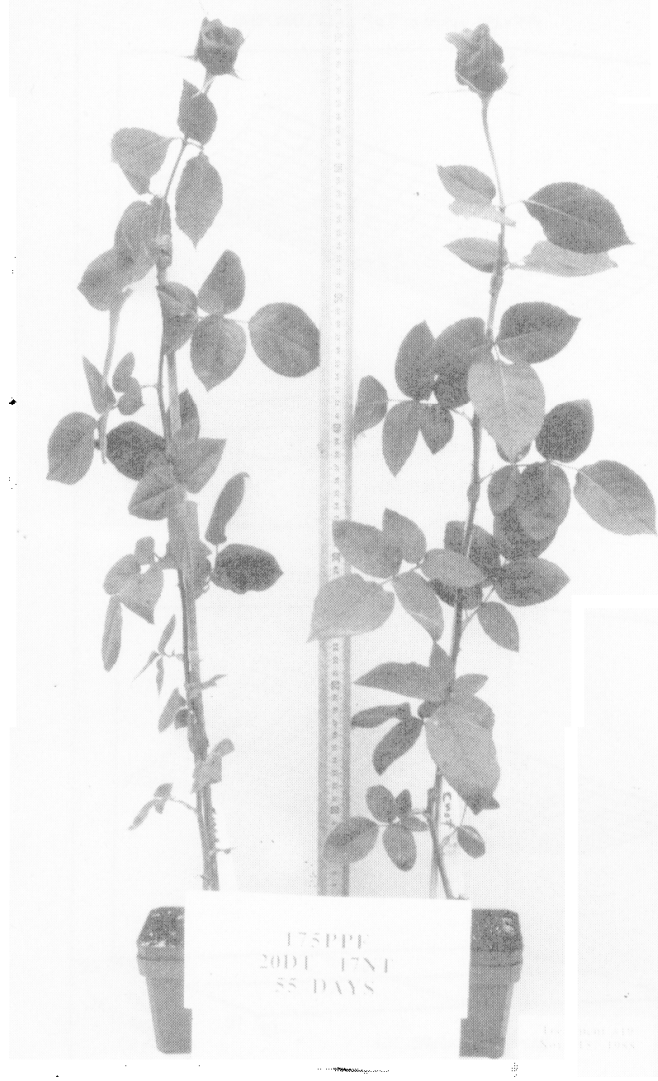

Fig. 3. Two representative rose plants from a repetition of the design center point (treatment $6: 175 \mu \mathrm{mol} \cdot \mathrm{m}^{-2} \cdot \mathrm{s}^{-1} \mathrm{PPF}, 20 \mathrm{C} \mathrm{DT}$, and $17 \mathrm{C}$ NT), showing flower size and quality at time of flowering.

predicted greater stem diameter over the design space as compared to the midpoint level of PPF.

Response surfaces were similar in shape for both fresh and dry weights of the stem, leaves, flower bud, and total flowering stem (Fig. 1B-I). All response surfaces were saddle shapes by mathematical definition, except for stem fresh weight, which was a minimum or bowl shape. However, the shapes of the surfaces are not readily apparent since the stationary points are located a considerable distance outside the design space (Table $5)$. The least fresh and dry weights for all plant parts occurred at the highest DT (25C) and highest NT (22C), while greatest fresh and dry weights occurred for the lowest combinations of DT (15C) and NT (12C) (Fig. 1B-I). Environments that promoted increased quality, as measured by greater stem diameter as well as greater fresh and dry weights, also retarded flower development. This was illustrated for all flower development criteria, including time from pinch until visible bud, first bud color, sepal reflex, and flower harvest (Fig. 1J-M). Under the constant environmental conditions provided from pinch until anthesis, time until anthesis was very nearly double the time until visible bud for all environments (Fig. $1 \mathrm{~J}$ and $\mathrm{M}$ ). This general rule would not be expected to apply precisely under greenhouse growing conditions, since seasonal weather changes would create different environments pre- and postvisible bud.

Armitage and Tsujita (1979a) concluded that increases in total fresh weight of flowers under supplemental PPF (158 $\mu \mathrm{mol} \cdot \mathrm{m}^{-2} \cdot \mathrm{s}^{-1}$ ) resulted in increased stem diameter; yet, our study revealed increased fresh and dry weights of stems, leaves, and flower buds, as well as increased stem diameter, under higher PPF $\left(250 \mu \mathrm{mol} \cdot \mathrm{m}^{-2} \cdot \mathrm{s}^{-1}\right)$. Although stem length has been observed to be reduced under supplemental PPF (Armitage and Tsujita, 1979a; Carpenter and Anderson, 1972; White and Richter, 1973), no significant differences for stem length were observed in our study (data not shown). Yield, as measured by the number of roses produced over time, has been shown to increase under supplemental PPF (Armitage and Tsujita, 1979a, 1979b; Butt and Tsujita, 1986; Carpenter and Anderson, 1972; Cockshull, 1975; Holcomb and Arteca, 1987; Tsujita, 1982; White and Richter, 1973), while Mattson and Widmer (1971) and Post and Howland (1946) found natural variation in solar radiation to similarly influence rose yield. In contrast, cultural methods in this study did not allow more than one flower to develop per plant, thereby limiting the observed yield. If yield is defined in terms of total carbon fixed into dry matter, then increased yield was observed when PPF was increased for this study as well. Irradiated rose plants in the greenhouse partition additional photosynthates into several sinks, thereby creating more flowering stems, each having little or no increases in weight (Armitage and Tsujita, 1979a; Holcomb and Arteca, 1987; Tsujita, 1982; White and Richter, 1973). In these instances, however, calculation of the quality index (QI $=$ yield $\times$ weight/ length), as described by Tsujita (1982), accounts for the significant increases in carbon fixed by the plants receiving greater PPF

Plants in our experiment increased in fresh and dry weights of stems, leaves, and flower buds under increased PPF (Fig. 2A-D), with no significant differences in partitioning between plant parts, unless shoots were blind, as under $50 \mu \mathrm{mol} \cdot \mathrm{m}^{-2} \cdot \mathrm{s}^{-1}$ PPF. This result contrasts with the findings of Butt and Tsujita (1986), where a greater percentage of both fresh and dry matter was partitioned to the stem as PPF increased. Increased PPF also reduced the number of days until visible bud and flower over the design space (Fig. 2E-H). In particular, of the plants grown under $50 \mu \mathrm{mol} \cdot \mathrm{m}^{-2} \cdot \mathrm{s}^{-1}$ in treatment 1 (Table 1 ), only one of the 10 plants grown to anthesis actually flowered; the remaining nine were blind, which indicates that PPF was indeed limiting in this lowest PPF treatment. Analyses conducted in this study used measurements from the single flowering plant as a representative mean for treatment 1 . This did not influence the statistical significance of the regression and canonical analyses since the analyses used the means of the treatments for deriving the sums of squares upon which statistical tests were conducted. Overall, the model $10-\mathrm{cm}$ pot system using singlestem plants produced flowers of size and quality similar to greenhouse production. Two representative plants from a repetition of the design center point illustrate the form and quality of typical flowers grown using these cultural techniques in growth chambers (Fig. 3).

Budbreak and anthesis of hybrid tea-rose seedlings has been reported to be delayed under low temperatures (DeVries et al., 1986). The delay of shoot growth and bud development to anthesis under low temperatures was confirmed with this study using 'Royalty'. However, the increased accumulation of fresh and dry weights in low DT and NT indicated quality was improved relative to higher temperatures. Hanan (1979) found that very low temperatures (4.4 and 10C) used in a split NT regime actually decreased quality, as measured by average stem length grade, but subsequently raising NT to $16.7 \mathrm{C}$ increased the yield of flowers with longer stems. Since the lowest DT and NT in this study were 15 and $12 \mathrm{C}$, respectively, the adverse effect of very low temperatures was not observed, while the increased dry matter accumulation by plants at 12 to $17 \mathrm{C}$ was evident as 
compared with the dry matter accumulation at temperatures of 20 to $25 \mathrm{C}$.

Quadratic regression models developed from the data allowed for a simple representation of plant response to the environment. These models apply specifically for constant environmental conditions from the time of pinching until anthesis. The general trends involving interactions between PPF, DT, and NT were conveniently represented as response surfaces that were easily interpretable. A more complex group of quadratic equations representing growth over 10-day periods should prove useful in formulating a dynamic model to predict growth over time under variable environments, such as in a greenhouse.

\section{Literature Cited}

Armitage, A.M. and M.J. Tsujita. 1979a. Supplemental lighting and nitrogen nutrition effects of yield and quality of Forever Yours roses.

Can. J. Plant Sci. 59:343-350.

Armitage, A.M. and M.J. Tsujita. 1979b. The effect of nitrogen concentration and supplemental light on the growth and quality of 'Caliente' roses. HortScience 14(5):614-615.

Armitage, A. M., W.H. Carlson, and C.E. Cress. 1981. Determination of flowering time and vegetative habit of Tagetes patula through response surface techniques. J. Amer. Soc. Hort. Sci. 106:632-638.

Box, G.E.P. and N.R. Draper. 1987. Empirical model-buiIding and response surfaces. Wiley, New York. p. 481-486.

Box, G. E. P., W.G. Hunter, and J.S. Hunter. 1978. Statistics for experimenters. Wiley, New York.

Butt, J. and M.J. Tsujita. 1986. Supplemental lighting. and carbon dioxide effects on Samantha rose growth and production. HortScience 21(3):278.

Carpenter, W.J. and G.A. Anderson. 1972. High intensity supplementary lighting increases yields of greenhouse roses. J. Amer. Soc. Hort. Sci. 97(3):331-334.

Cochran, W.G. and G.M. Cox. 1957. Experimental designs. 2nd ed. Wiley, New York. p. 344-346.

Cockshull, K.E. 1975. Roses II: The effects of supplementary light on winter bloom production. J. Hort. Sci. 50:193-206.
DeVries, D. P., L.A.M. Dubois, and L. Smeets. 1986. The effect of temperature on axillary bud-break of hybrid tea-rose seedlings. Scientia Hort. 28:281-287.

Hammer, P.A. and R.W. Langhans. 1976. Growth models for $\mathrm{He}$ lianthus annuus L. and Zinnia elegans. Jacq. J. Amer. Soc. Hort. Sci. 101:475-479.

Hanan, J.J. 1979. Observation of a low temperature effect on roses. J. Amer. Soc. Hort. Sci. 104(1):37-40.

Heins, R. D., M.G. Karlsson, J.A. Flore, and W.H. Carlson. 1986. Effects of photosynthetic rate maximization on chrysanthemum growth and development. J. Amer. Soc. Hort. Sci. 111:42-46.

Holcomb, E.J. and R. Arteca. 1987. Yield and photosynthesis of roses grown under varying light-temperature regimes. Roses Inc. Bul. August: 97-106.

Jaio, J., M.J. Tsujita, and R.G. Dutton. 1988. Phase I-Environmental parameters for computer-controlled greenhouses; Phases II-Temperature, for roses as influenced by light levels. Roses Inc. Bul. January: 83-93.

Kraszewski, R.A. and D.P. Ormrod. 1986. Utilization of a response surface technique to study light acclimation of indoor flowering plants. J. Amer. Soc. Hort. Sci. 111(1):47-55.

Mattson, H.R. and R.E. Widmer. 1971. Effects of solar radiation, carbon dioxide, and soil fertilization on Rosa hybrida. J. Amer. Soc. Hort. Sci. 96(4):484-486.

Moe, R. 1971. Factors affecting flower bud abortion and malformation in roses. Plant Physiol. 24:291-300.

Myers, R.H. 1976. Response surface methodology. Virginia Polytechnic Inst. and State Univ., Blacksburg.

Post, K. and J.E. Howland. 1946. The influence of nitrate and light intensity on the growth and production of greenhouse roses. Proc. Amer. Soc. Hort. Sci. 47:446-450.

Savage, M.J. 1982. Radiation in plant research: A plea for standardization. HortScience 17(2):134-135.

Tsujita, M.J. 1982. Flower cutting practices for greenhouse roses receiving supplemental irradiation. HortScience 17(1):44-45.

White, J.W. and D. Richter. 1973. Supplementary fluorescent lighting and low moisture stress improve growth of greenhouse roses, J. Amer. Soc. Hort. Sci. 98(6):605-607. 